
Revue Revue de l'histoire des religions
de Ihistoire des religions
$2 \mid 2020$
Ce qu'un dieu n'est pas : les prémices de la théologie négative

\title{
Ce qu'un dieu n'est pas : les prémices de la théologie négative
}

Avant-propos

Stéphanie Anthonioz et Sebastian Fink

\section{OpenEdition \\ Journals}

Édition électronique

URL : https://journals.openedition.org/rhr/10506

DOI : 10.4000/rhr.10506

ISSN : 2105-2573

Éditeur

Armand Colin

\section{Édition imprimée}

Date de publication : 1 juin 2020

Pagination : 179-193

ISBN : 978-2-200-93326-5

ISSN : 0035-1423

Référence électronique

Stéphanie Anthonioz et Sebastian Fink, «Ce qu'un dieu n'est pas : les prémices de la théologie négative », Revue de l'histoire des religions [En ligne], 2 | 2020, mis en ligne le 01 janvier 2021, consulté le 03 janvier 2023. URL : http://journals.openedition.org/rhr/10506 ; DOI : https://doi.org/10.4000/rhr 10506 


\section{Ce qu'un dieu n'est pas : les prémices de la théologie négative Avant-propos}

La théologie est un discours dont l'objet d'étude porte sur le divin, le ou les dieux. Elle est un concept né en Grèce à l'époque classique et trouve son origine dans un dialogue de Platon (La République II, 379 a - 383 c). Or l'objet de ce discours n'est pas comme les autres, puisqu'il s'agit d'une transcendance dont on pressent rapidement qu'elle est susceptible de dépasser tant la compréhension rationnelle qu'on peut s'en faire que les catégories du langage qui tentent de l'exprimer. Le ou les dieux semblent, pour une large part, échapper à l'entreprise humaine dans sa visée épistémologique et représentationnelle. Comment, en effet, pourrait-on exprimer au sein d'un discours ou encore figurer en images ce qui semble d'emblée les outrepasser par sa réalité même. Le ou les dieux déborde(nt) le cadre limité et fini tant de la conceptualisation que de la représentation. C'est à l'examen de cette question que ce collectif sur « la théologie négative » va contribuer ${ }^{1}$. En effet, l'une des solutions qui a vu le jour consiste en l'émergence d'une théologie négative : à défaut de

1. Nous souhaitons vivement remercier la Revue de l'histoire des religions qui a permis la réalisation de ce collectif et a contribué grandement à l'enrichir par le travail de relecture critique. Nous souhaitons également remercier notre collègue Pascaline Turpin à Lille qui n'a pas hésité à éclairer le concept de théologie négative de son point de vue de philosophe. 
pouvoir dire ce que Dieu ou les dieux sont, on dira ce qu'il n'est et ce qu'ils ne sont pas. Seront plus particulièrement prises en charge les prémices d'une telle théologie dans les religions anciennes. Pourquoi et comment dire le divin par l'entremise d'un "ne pas », négation linguistique par excellence, puisque c'est de fait encore discourir sur le divin?

\section{LA THÉOlOGIE NÉGATIVE DANS LES SOURCES PHILOSOPHIQUES, PATRISTIQUES ET MÉDIÉVALES}

Qu'est-ce que la théologie négative ? Il s'agit avant tout d'un discours sur Dieu ou les dieux, qui use de la négation linguistique dans le but d'en manifester l'éminence, voire la suréminence. C'est aussi une manière de montrer l'insuffisance du langage pour dire Dieu ou les dieux sans pour autant y renoncer. Il est admis que les sources de cette théologie sont à rechercher dans les dialogues de Platon, particulièrement le Parménide (141-142), puis dans les écoles platoniciennes, dont on connaît la profonde influence sur la pensée chrétienne.

Dans le cadre de cet avant-propos, il importe d'emblée de souligner une évolution pertinente de la théologie négative, celle de la «coïncidence des contraires ». En effet, au tout début du $\mathrm{VI}^{\mathrm{e}}$ siècle, l'une des voies préconisées par le Pseudo-Denys pour avancer dans une telle démarche négative est la dialectique: les notions tirées de l'expérience sont appliquées au divin et regroupées par couples antinomiques. De cette manière, les concepts se corrigent mutuellement, et l'esprit peut échapper à la tentation de réduire dans un savoir clos la transcendance divine ${ }^{2}$. Plus tard, Nicolas de Cues (1401-1461) proposera le concept de coincidencia oppositorum ( "coïncidence des contraires»), soulignant par son biais le fait que deux propositions contraires au sujet de Dieu peuvent être également vraies ${ }^{3}$. Il serait donc

2. Le chapitre IX des « Noms divins » illustre la méthode par quatre associations de concepts : grandeur / petitesse, identité / altérité, similitude / différence, stabilité / mobilité. Voir C. Wackenheim, « Actualité de la théologie négative », Revue des Sciences Religieuses, 59, 1985, p. 147-161, ici p. 152.

3. À comparer au livre des vingt-quatre philosophes (Liber XXIV philosophorum), dans lequel on peut trouver la proposition Deus est sphaera infinita cuius centrum est ubique, circumferentia nusquam. 
nécessaire de dépasser le principe de non-contradiction ${ }^{4}$, lorsque l'on veut approfondir la connaissance divine. En même temps, le projet d'une telle théologie peut paraître paradoxal. Car, malgré les insuffisances de la démarche discursive, c'est celle-là même qui est adoptée pour exercer le jugement. La théologie négative implique donc (et développe) une pratique féconde que C. Wackenheim appelle «l'écart épistémologique et herméneutique $»^{5}$, qui pointe, en dernier ressort, vers les limites de toute représentation du divin, qui sont bien les limites humaines pour penser le divin. Aussi peut-on également parler d'une théologie apophatique. Car

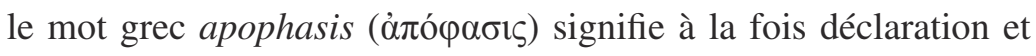
négation. La théologie négative ou apophatique inscrit alors ses affirmations dans un champ borné par des certitudes négatives ${ }^{6}$. Il importait de souligner le développement historique de ce concept théologique, puisque plusieurs contributions dans ce collectif mettent en évidence une telle « coïncidence des contraires », dans les sources anciennes, même si bien sûr elle n'est jamais systématisée comme telle.

Dans tous les cas, il apparaîtra que la via negationis ou via negativa - selon la terminologie anglo-saxonne-, qu'elle soit conceptualisée ou non, qu'elle soit systématisée ou non, apparaît comme le corollaire d'autres voies possibles pour penser et représenter le ou les dieu(x).

\section{QUELLES SONT LES MANIÈRES DE PENSER ET REPRÉSENTER LE DIVIN DANS L'ANTIQUITÉ DU BASSIN MÉDITERRANÉEN ET DU GOLFE PERSIQUE?}

Les différentes cultures du bassin méditerranéen et du golfe persique dans l'Antiquité n'ont cessé de penser et de représenter le divin. Ces représentations épousent des formes aussi nombreuses que diversifiées, visuelles ou conceptuelles. Elles ne sont pas exclusives et cohabitent généralement assez facilement. Il paraît

4. C'est-à-dire qu'il est impossible, selon Aristote, Métaphysique (livre Gamma), pour un sujet de recevoir deux attributs contradictoires en même temps et sous le même rapport.

5. C. Wackenheim, «Actualité de la théologie négative », p. 156.

6. C. Wackenheim, «Actualité de la théologie négative », p. 147-161. 
opportun de les rappeler. Cette synthèse préalable permettra d'envisager comment la question de la théologie négative se pose dans différentes cultures et ainsi d'introduire aux contributions de ce collectif qui couvrent certaines aires géographiques du bassin méditerranéen et du golfe Persique.

Il ne s'agit pas ici - ce qui serait impossible - de présenter un examen exhaustif des différentes manières de penser et représenter le divin ${ }^{7}$. Il paraît suffisant de recourir à quelques concepts qui permettent de visualiser et de mesurer sa présence diffuse et diversifiée dans les cultures antiques, car les représentations, qu'elles soient visuelles ou conceptuelles, peuvent être humaines, animales, astrales, végétales ou encore minérales. Ces modes de représentation semblent être partagés, indépendamment des questions d'influences culturelles, d'où l'intention ici de ne pas réduire à une aire géographique unique même si l'examen de fait est limité, général et ne peut donner lieu qu'à des particularités locales.

Les représentations astrales en milieu mésopotamien ont été notamment étudiées par Beate Pongratz-Leisten ${ }^{8}$. Mais il convient de souligner que l'auteure commence son étude en rappelant un principe que l'on pourrait par commodité appeler « sémitique », à savoir que les anciens ne distinguent pas le corps de la personne. Au contraire des traditions plus tardives, platoniciennes et cartésiennes,

7. Comme l'a redit Karel Van der Toorn, «Speaking of Gods: Dimensions of the Divine in the Ancient Near East », Open-Mindedness in the Bible and Beyond: A Volume of Studies in Honour of Bob Becking, dir. Marjo Korpel, Lester L. Grabbe, London, T\&T Clark, 2015, p. 275 : «A brief survey of all the elements that belong to the class of 'gods' (DINGIR) yields an impressive list. Let us for the moment ignore chronological variations and try to arrange the various gods in subgroups. The divine classifier is applied to cosmic phenomena such as Heaven, Air, Earth, Moon, Sun, Stars; natural phenomena such as mountains, rivers and seas; extraordinary human beings, in particular kings and the dead; objects that pertain to the sphere of the gods and their cult such as temples, divine images and symbols, cultic paraphernalia; particular topographic places, such as Ashur, originally a so-called numen loci; and some rare abstractions such as Tashmetu, 'Concord,' and Duru and Daru, 'Time-and-Eternity'; and finally a variety of beings that by our standards would qualify as demons, angels, and demi-gods. »

8. B. Pongratz-Leisten, "Divine Agency and Astralization of the Gods in Ancient Mesopotamia », Reconsidering the Concept of Revolutionary Monotheism, dir. B. Pongratz-Leisten, Winona Lake, Eisenbrauns, 2011, p. 137-187. Voir également Joan G. Westenholz, "The Body and the Mind in Mesopotamian Traditions », Menschenbilder und Körperkonzepte im Alten Israel, in Ägypten und im Alten Orient, dir. Angelika Berlejung et alii, Tübingen, Mohr Siebeck («Orientalische Religionen in der Antike», 9), 2012, p. 459-477. 
d'une dichotomie entre un corps anatomique, matériel, spatial et temporel mais surtout fragile et un esprit intellectuel, spirituel, universel et infaillible, Sumériens et Babyloniens saisissent la personne comme l'assemblage de ses parties, à savoir le corps, mais aussi ses noms, ses fonctions et ses images. Aussi le corps fait-il partie de la définition de l'être. Il n'y a aucune séparation entre physique et psychique au point que certaines parties corporelles sont responsables de certaines fonctions cognitives et peuvent représenter symboliquement la personne ${ }^{9}$. Or cette conception s'applique aussi au monde divin, tout en s'élargissant : les dieux sont présents et agissent à travers leurs représentations cultuelles, leurs lieux, leurs attributs :

In the ancient Near East, the notion of the "divine" is similarly not limited to an anthropomorphic deity. Rather than conceptualizing the divine and the human worlds as distinct realms, the human sphere gradually merges with the realm of the divine. Thus living kings, dead kings, and ancestors can all be said to have divine quality. Interaction with the major gods might occur through objects connected with their cult, among them the cult statue, the gods' standards, emblems, weapons, tiaras, thrones, chariots and boats used during the procession, steles and abstract concepts, all of which might be anthropomorphized in the sense of being assigned agency and which might, consequently, function as the secondary agents of a deity. ${ }^{10}$

L'exemple le plus célèbre dans l'Antiquité du Proche-Orient est certainement la tablette dite de Šamaš, dieu Soleil, retrouvée dans son temple Ebabbar, à Sippar, antique ville babylonienne (BBSt $36)^{11}$. Trouvée à l'époque du roi Nabopolassar (625-605) déjà

9. Les reins sont, par exemple, le lieu du discernement, le cœur mais aussi les oreilles, celui de l'intelligence. Les yeux dans leur transparence peuvent être associés, par contre, à différents sentiments. Les narines peuvent signifier la colère comme la langue ou la bouche, la calomnie. Les mains, les bras et les jambes désignent souvent la force ou la puissance.

10. B. Pongratz-Leisten, «Divine Agency », p. 143. Voir aussi Gebhard Selz, " "The Holy Drum, the Spear, and the Harp:" Towards an Understanding of the Problems of Deification in Third Millennium Mesopotamia », Sumerian Gods and Their Representations, dir. Markham J. Geller, Irving L. Finkel, Groningen, Styx Publications («Cuneiform Monographs », 7), 1997, p. 149-194.

11. Voir Matthias Köckert, "Suffering from Formlessness: The Ban on Images in Exilic Times », Exile and Suffering: A Selection of Papers Read at the $50^{\text {th }}$ Anniversary Meeting of the Old Testament Society of South Africa OTWSA/ OTSSA, Pretoria august 2007, Leiden, Brill, 2009, p. 33-49 et particulièrement p. 43-45 ; Christopher E. Woods, "The Sun-God Tablet of Nabû-apla-iddina Revisited», Journal of Cuneiform Studies, 56, 2004, p. 23-103; Victor A. Hurowitz, "The Sun-Disk Tablet of Nebobaladan, King of Babylon (BBSt 
cassée, elle fut remplacée tandis que les morceaux étaient disposés dans un coffre en terre cuite avec d'autres articles et enterrés sous le sol du temple. Mais le respect qu'elle avait fait naître était lié à une antiquité plus haute, puisque l'inscription raconte comment le roi babylonien Nabu-aplu-iddina (888-855), en l'absence de la statue cultuelle, exposa dans l'attente de sa restauration le symbole que la tablette représente, l'astre solaire guidé par d'autres divinités ou génies.

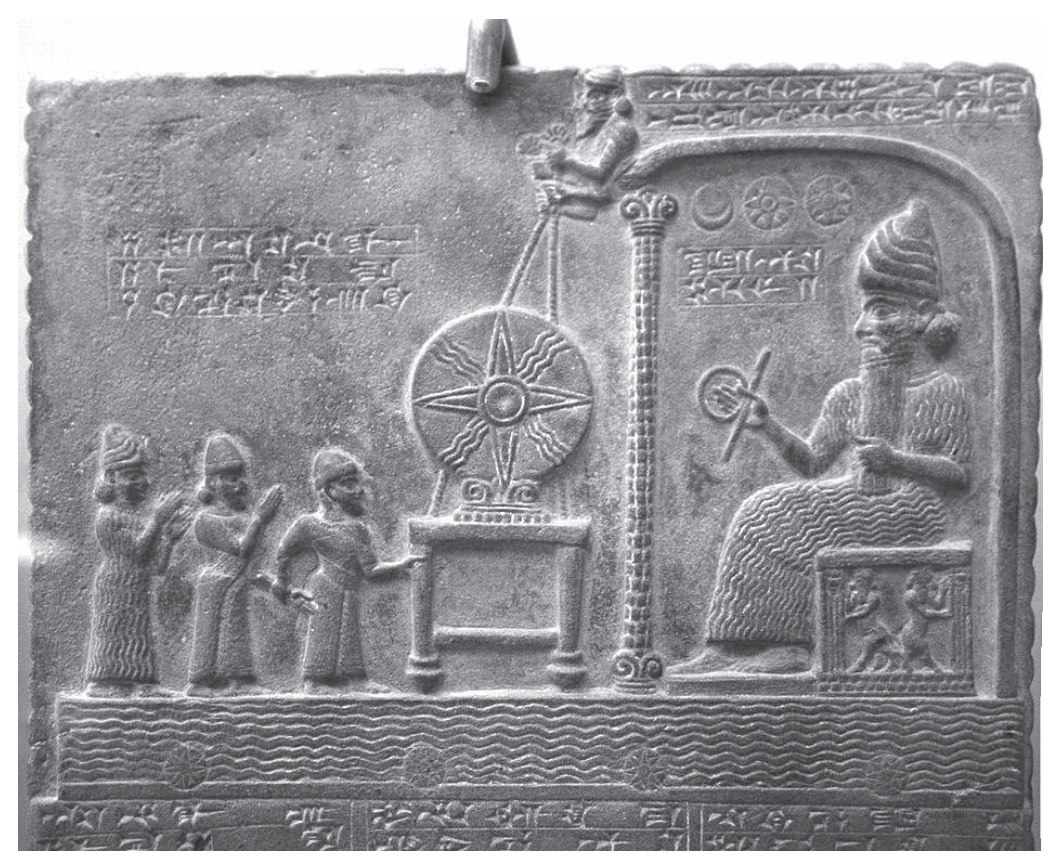

Relief de la tablette de Šamaš, British Museum, salle 55 (ME 91000). Commons.wikimedia.org

L'exemple de la tablette de Šamaš permet de comprendre que toutes les représentations ne sont pas identiques, mais que chacune est porteuse d'une puissance de la divinité qu'elle représente, ce que B. Pongratz-Leisten propose d'appeler divine agency. La divinité est ainsi nommée "puissance première », tandis que ses différentes représentations, c'est-à-dire les statues, les symboles ou les astres,

36) », Eretz Israel, 27, 2003, p. 91-109 ; Kathryn E. Slanski, «Classification, Historiography and Monumental Authority: The Babylonian Entitlement narûs (kudurrus) », Journal of Cuneiform Studies, 52, 2000, p. 95-114. 
sont des «puissances secondaires $»^{12}$. La réflexion que l'auteure poursuit invite à saisir le rapport entre ces deux types. Aussi les puissances secondaires ne sont pas dotées de la même manière que les puissances premières, mais représentent plutôt une fonction particulière. Et le nom divin, plutôt que d'évoquer une personnalité divine, pointerait vers une efficacité déterminée. La diversité des représentations divines, qui est aussi fluidité, n'empêche pas les anciens de penser l'unité, comme le nom personnel Gabbu-ilāniAššur, «Aššur-est-tous-les-dieux », le démontre, nom qui apparaît dans les sources vers le VIII siècle av. n. è. et que l'on connaît abondamment dans les textes babyloniens mystiques où Marduk est $\ll$ tous les dieux $»^{13}$.

Clairement la présence divine n'est pas une mais diffuse autant que diversifiée à travers l'ensemble de ses représentations. L'anthropomorphisme appartient ainsi au monde des représentations du divin parmi d'autres représentations majeures, animales, astrales ou minérales. Cette matérialité ou «matérialisation» est diversement documentée, outre les sources textuelles, par les images, les objets issus des fouilles et des sites archéologiques. Il suffit de citer deux contributions au titre évocateur de l'auteur Izak Cornelius, The Many Faces of the Goddess, d'une part, et «The Many Faces of God : Divine Images and Symbols in Ancient Near Eastern Religions », d'autre part ${ }^{14}$.

12. B. Pongratz-Leisten, « Divine Agency », p. 146. Plus récemment Michael B. Hundley, Gods in Dwellings: Divine Presence in the Ancient Near East, Atlanta, SBL, 2013, a enquêté sur la présence et les représentations du divin dans le monde antique, en particulier les modes d'inhabitation des temples en Égypte, Mésopotamie, Anatolie hittite et Syrie-Palestine. L'auteur montre comment la divinité habite son temple souvent sous la forme d'une statue cultuelle et revient sur le rapport entre la divinité et son image, le rapport entre la divinité et ses autres formes de représentation. Voir bientôt les travaux de S. Fink qui propose pour sa part de recourir à la notion de «perspective» dans le sens exploré par Leibniz, La Monadologie ( «t comme une même ville regardée de différents côtés paraît toute autre et est comme multipliée perspectivement, il arrive de même que, par la multitude infinie des substances simples, il y a comme autant de différents univers, qui ne sont pourtant que les perspectives d'un seul selon les différents points de vue de chaque monade. » $\$ 57$ ).

13. Notion développée par Mark S. Smith, God in Translation: Deities in Cross-Cultural Discourse in the Biblical World, Tübingen, Mohr Siebeck («Forschungen zum Alten Testament», 57), 2008, p.168s.

14. I. Cornelius, The Many Faces of the Goddess: The Iconography of the Syro-Palestinian Goddesses Anat, Astarte, Qedeshet, and Asherah c. 1500-1000 $B C E$, Fribourg - Göttingen, Academic Press Fribourg - Vandenhoeck \& Ruprecht 
De notre point de vue, qui vise à offrir une brève synthèse afin de mieux introduire à la question des prémices de la théologie négative, on peut, en regard de la matérialité, souligner les choix représentatifs et fonctionnels de telle ou telle divinité. Šamaš, dieu Soleil par exemple, n'est jamais représenté par la planète Vénus. Cette simple remarque permet déjà de mesurer la part de négation dans le choix représentatif et les limites intrinsèques du nom divin, de ses représentations et de ses fonctions: une divinité est ce qu'elle est dans la diversité de ses noms, attributs et représentations, certes, mais dans la limite de ses fonctions. Elle n'est donc pas autre chose. Cette particularité qu'implique toute matérialité appartient au langage, qu'il soit visuel ou conceptuel ; elle nous permet maintenant d'appréhender de manière plus détaillée ce que l'on entend, dans les sources antiques, par «prémices » de la théologie négative.

\section{CE QUE L'ON ENTEND PAR « PRÉMICES » DE LA THÉOLOGIE NÉGATIVE DANS L'ANTIQUité}

Nous ne ferons que poser quelques jalons. Sebastian Fink a proposé de distinguer au moins deux strates d'une évolution religieuses dans les sources cunéiformes ${ }^{15}$. Une première strate serait celle des mythes et des épopées dans laquelle les dieux sont représentés de manière anthropomorphique : les dieux sont en relation avec les humains, les séduisent parfois, ressentent des émotions fortes. Par ailleurs, ils habitent des maisons, ils mangent et boivent. Ainsi, comme dans la tradition épique et mythologique grecque, les dieux sont pensés à la manière des êtres humains malgré des pouvoirs surnaturels et souvent la vie éternelle. Pourtant, si l'on se tourne vers le corpus des prières et, plus particulièrement, celui des Lamentations, c'est une autre conception du divin qui se fait jour, où les dieux deviennent plus

(«Orbis Biblicus et Orientalis », 204), 2004 ; id., « The Many Faces of God: Divine Images and Symbols in Ancient Near Eastern Religions », The Image and the Book, dir. Karel van der Toorn, Leuven, Peeters, 1997, p. 21-43.

15. S. Fink, «Metaphors for the Unrecognizability of God in Balags and Xenophanes », Mesopotamia in the Ancient World: Impact, Continuities, Parallels, dir. Robert Rollinger, Erik van Dongen, Münster, Ugarit-Verlag (« Melammu Symposia », 7), 2015, p. 231-243. 
distants, plus transcendants. La collection, en langue emesal (variante du sumérien), des Lamentations Balaĝs est documentée depuis le début du $\mathrm{II}^{\mathrm{e}}$ millénaire ${ }^{16}$. Elle a été recopiée jusqu'à la fin de l'ère cunéiforme, les copies les plus tardives pouvant être datées au dernier siècle av. n. è. Cette collection a été mise par écrit à une époque où le sumérien n'était déjà plus parlé et laisse penser que le genre appartenait à la tradition orale. Les spéculations théologiques et étymographiques des scribes - peutêtre également d'origine orale - sont documentées en akkadien dans des traductions interlinéaires, certaines étant parfaitement claires, d'autres hautement ésotériques concernant les messages secrets du «texte sacré sumérien $»^{17}$. Les copies en quantité montrent l'importance du genre et leur récitation régulière, hebdomadaire voire quotidienne ${ }^{18}$, est attestée à l'époque néo-assyrienne, c'est-à-dire tout au long de la première moitié du premier millénaire av. n. è.

Si l'on s'intéresse maintenant aux prémices de la théologie négative dans la collection des Balaĝs, il est nécessaire de faire un détour par les célèbres inscriptions du roi Gudéa (fin du III ${ }^{e}$ millénaire). Dans le Cylindre B, VIII, 48, il est dit que le nom $\mathrm{du}$ dieu Suen ne peut être résolu ( ${ }^{d}$ suen-mu-ni $l u_{2} n u-d u_{8}-d e_{3}$ ), littéralement «ouvert» (sum. $\left.d u_{8}\right)$. Manfred Krebernik estime qu'il s'agit d'exprimer l'étymologie peu claire du nom Suen ${ }^{19}$. En effet, on sait que le nom révèle l'identité de son propriétaire et, si l'interprétation de l'expression est juste, alors on peut dire qu'au III $^{\mathrm{e}}$ millénaire av. n. è. déjà les scribes tentent de comprendre le divin par la voie de l'étymologie et de l'interprétation du nom. Mais si le nom divin ne peut pas être compris et s'il reste obscur,

16. Mark E. Cohen, The Canonical Lamentations of the Ancient Mesopotamia. 2 Vol., Potomac, Maryland, 1988.

17. Stefan Maul, «Küchensumerisch oderhohe Kunst derExegese? Überlegungen zur Bewertung akkadischer Interlinearübersetzungen von Emesal-Texten », Ana šad $\hat{\imath}$ Labnāni lū allik: Beiträge zu altorientalischen und mittelmeerischen Kulturen: Festschrift für Wolfgang Röllig, dir. B. Pongratz-Leisten et alii, Neukirchen-Vluyn («Alter Orient und Altes Testament», 247), 1997, p. 253-267.

18. Pratique documentée dans les manuels des chantres, comme on le voit dans les textes 12-14 édités par Simo Parpola, Assyrian Royal Rituals and Cultic Texts, Helsinki, The Neo-Assyrian text corpus project (« State archives of Assyria », 20), 2017.

19. M. Krebernik, «Mondgott. A. I. in Mesopotamien», Reallexikon der Assyriologie, 8/5-6, 1995, p. 360-369, ici p. 362. 
alors la nature divine doit demeurer mystérieuse. Or ce thème de l'interprétation du nom est aussi présent dans le Balag intitulé Úru Àm-ma-ir-ra-bi (tablette 21, ligne 89), où il est dit que les prêtres ne peuvent pas expliquer les noms choisis de la déesse Inanna / Ištar, faisant ainsi référence à ses épithètes ${ }^{20}$. Ces deux exemples mettent en lumière l'incapacité humaine à s'emparer du divin. Plus encore, les dieux ne se comportent pas comme dans les mythes ou les épopées, de manière humaine. En effet, les Lamentations évitent de les mettre en scène et de leur attribuer une action, souvent d'ailleurs une destruction. Une instance est donc déléguée à ces «affaires » et c'est la fonction typique de la parole ou du commandement divin. La divinité ne réalise pas la destruction mais son ordre le fait. Cette tradition est déjà attestée dans le recueil des Lamentations des villes au nombre de cinq, recueil qui a joui d'une autorité à la fin de l'empire d'Ur III et dont la tradition des Balaĝs n'est certainement pas indépendante. Ainsi, dans la Lamentation d'Ur, Enlil appelle ( $\left.g u_{3}-d e_{2}, \mathrm{LU} 173\right)$ le(s) « jour(s) de tempête » (1. 173). Cette dernière est personnifiée et agit comme arme d'Enlil :

197 (Cette) tempête mauvaise, ce n'est pas la compassion qui fixe (son) destin - le peuple gémit.

198 La tempête qui rase (tout) nivelle le pays.

199 La tempête : telle un déluge, elle détruit de fond en comble les villes.

200 La tempête qui anéantit tous les pays plongea la ville dans le silence,

201 la tempête qui efface tout se joignit aux forces maléfiques,

202 la tempête qui brûle comme le feu déchira les chairs des gens,

203 la tempête haineuse ordonnée par Enlil, la tempête qui affaiblit le pays

204 recouvrit Ur tel un vêtement, la drapa telle une étoffe de lin. ${ }^{21}$

Dans les Lamentations Balaĝs, la «tempête » est souvent remplacée par la « parole divine ${ }^{22}$, sauf en quelques endroits où elle lui est assimilée :

20. Konrad Volk, Die Balag-Komposition Úru Àm-ma-ir-ra-bi, Freiburg (« Freiburger Altorientalische Studien », 18), 1989, p. 206.

21. Pascal Attinger, «La lamentation sur Ur (2.2.2)», disponible en ligne sur www.iaw.unibe.ch/e39448/e99428/e122665/e122821/pane122850/e122909/ 2_2_2.pdf

22. Voir Sebastian Fink et Mark S. Smith, « The Day-Storm in Mesopotamian Literature: A Background to the Biblical Day of Yahweh? »(en préparation). 
88 Sa parole (est) la tempête du sudd ${ }^{23}$, la tempête du sud ... le pays. 89 Clameur et malheur ! Ma ville !... Malheur !

90 La parole d'Enki (est) la tempête du sud... le pays.

‥

96 La parole de Dikumaham (est) la tempête du sud ... le pays. ${ }^{24}$

Ce procédé relève sans doute du langage métaphorique, à ceci près que, dans le même texte, l'ordre divin ne peut être soumis à l'interprétation humaine, pas même à celle des devins qui risqueraient alors une parole mensongère. L'incapacité humaine à comprendre le divin est assénée au moyen de longues litanies, qui se concluent par la question rhétorique mu.lu ta.zu ти.ип.zu - "Que peut-on savoir à ton sujet?». Les Lamentations regroupent aussi un certain nombre de mises en garde contre l'anthropomorphisme divin. Elles insistent sur le fait que les dieux ne marchent pas comme les hommes (88. umun kur-kur-ra mu-lu til-la-gin ${ }_{7}$ na-ra-[di-di-in] 89. $\check{s} a_{3}-m u$ ma-al-la-gin ${ }_{7}$ na-ra-[di-di-in], «Seigneur des pays, tu ne te promènes pas comme un humain. Tu ne te promènes pas comme je le ferais $\gg)^{25}$ ou encore qu'ils ne naissent pas comme les hommes (35. alim-ma pap-hal nu-nuz-ke ${ }_{4} n u-e_{3}-a-z u-d e_{3}$, «Bison, puisque tu n'es pas né du ventre d'une femme, que peut-on savoir à ton sujet ? » $)^{26}$ : c'est ici que les prémices d'une théologie négative sont identifiées. Elles se trouvent au côté d'autres outils stylistiques, la question rhétorique et la métaphorisation. Le discours utilise donc également la voie positive, la voie interrogative et la négation. Certainement ces recours diversifiés pointent vers les limites du langage et un certain agnosticisme, à savoir la conscience des limites humaines et des limites des outils humains pour appréhender et comprendre le divin. Et il ne s'agit évidemment pas d'un système métaphysique, au sein duquel on comprendrait l'utilisation de la négation comme indice ou signe de la transcendance. Mais il n'en demeure pas moins que la via negationis, comme exploration nouvelle, révèle une inadéquation entre le sujet pensant et l'objet pensé, bien attestée dans ces sources d'une haute antiquité.

23. Alors qu'un vent du sud peut être considéré comme une brise agréable dans les contrées du nord, il est, au contraire, considéré comme particulièrement destructif dans une région chaude comme la Mésopotamie. Merci à Izaak de Hulster pour cette réflexion.

24. M. E. Cohen, The Canonical Lamentations, p. 78-87.

25. Ibid., p. 258.

26. Ibid., p. 459. 


\section{LES CONTRIBUTIONS PROPOSÉES DANS CE COLLECTIF}

Il est important de souligner que si l'histoire de la théologie négative est devenue philosophique, c'est-à-dire purement conceptuelle, les représentations du divin dans l'Antiquité associent étroitement les dieux et la diversité de leurs matérialisations. Il ne conviendrait donc pas, au regard des sources, de se limiter aux textes et certaines contributions qui suivent font la part belle à la dimension visuelle et matérielle de la représentation des dieux.

Le collectif est composé d'une contribution consacrée à la Mésopotamie, de deux contributions consacrées au Levant, et deux autres à la Grèce. Une dernière contribution revient sur les sources bibliques. Dans une dimension historique, elle accorde une place importante aux images et se voit donc particulièrement bien éclairée par les réflexions grecques et philosophiques qui la précèdent. Cette architecture de l'ensemble permet ainsi de mettre en lumière les évolutions et les influences possibles au sein du bassin méditerranéen dans la deuxième partie du Ir millénaire av. n. è.

Peeter Espak, "What is not God. The Earliest Sources in Comparative Perspective », nous invite à considérer les sources cunéiformes antiques de Mésopotamie. L'analyse de la documentation suméro-akkadienne, datée du III ${ }^{\mathrm{e}}$ millénaire av. n. è., démontre l'immanence des divinités, qui bien que distinctes de tout autre créature, habitent le cosmos et ne sont pas des créations secondes. Les divinités peuvent ainsi être considérées comme une forme d'existence permanente dans le cosmos, tandis que toutes les autres créatures, humaines ou animales, sont des développements secondaires. Pour autant cette distinction ne permet pas, selon l'auteur, d'élaborer quelque théologie négative, même si un certain nombre de tensions (humain / inhumain ; mortel / immortel ; généré / créé) marquent les limites d'une représentation conceptuelle du divin. L'apport de cette contribution réside dans l'exposition d'un système religieux dans lequel les dieux sont à l'image des humains, sauf sur un point précis, l'origine.

Fabio Porzia, «Noms de dieux et théologie négative au Levant dans l'Antiquité », propose de partir de l'étude des noms de dieux, qui donne accès à un véritable savoir théologique. L'article se concentre sur des noms parlants qui ont parfois fait penser à des dieux sans nom propre, voire anonymes. En inscrivant ces noms 
dans le système de nomination sémitique, cet article montre que, d'une part, il est inexact de considérer ces divinités comme anonymes, mais que, d'autre part, les noms de dieux formés sur les noms communs de ba'al, 'el, 'adon, melek, par exemple, ont pu ouvrir aux développements successifs de la théologie négative.

Annette Schellenberg, "Traces of Negative Theology in the Hebrew Bible », propose un éventail de cas relevant manifestement d'une théologie négative dans la Bible hébraïque : l'interdiction des images divines, le récit que l'on peut appeler de la révélation du nom («je suis qui je suis», Ex 3,13), celui ne permettant à Moïse que de contempler Dieu par derrière (Ex 33), ou encore la narration concernant Élie au mont Horeb (1 R 19), la tradition de l'incomparabilité divine, l'incapacité humaine à discerner, enfin la juxtaposition de différentes métaphores au sujet de Dieu. Ces textes sans renier la possibilité d'énoncés positifs, soulignent l'inadéquation de ces énoncés, d'une part, et, d'autre part, l'incapacité humaine à pleinement saisir Dieu.

Alberto Bernabé, «Théologie négative dans la pensée présocratique », met en lumière les principaux témoignages présocratiques qui permettent de dresser une première histoire de la théologie négative. Plusieurs développements sont identifiés chez les présocratiques qui viennent contredire en partie les idées concernant les dieux, véhiculées par la tradition grecque. Parmi ceux-ci, Anaximandre, en attribuant à une réalité naturelle - le

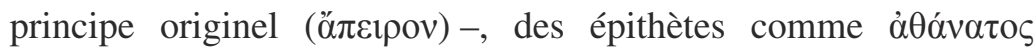
et ảy indiquer que ce qui mérite d'avoir la condition de dieu est cette nouvelle réalité originelle qu'il postule. Xénophane, pour sa part, insiste sur les inconséquences de l'image homérique des dieux et critique explicitement ce qu'il considère ne pas être le propre d'un dieu. Il développe ainsi une théologie négative en parallèle à une théologie positive. Mais la contradiction esquissée entre la vision traditionnelle des dieux et la vision qu'en a la philosophie doit encore être considérée du point de vue du culte. Quelle que soit la nature du postulat d'un dieu philosophique, les dieux ne sont pas dieux, si un culte ne peut leur être rendu. C'est ainsi la diversité des points de vue autant que les développements de la philosophie négative chez les présocratiques que cette contribution éclaire de manière fascinante. 
Pablo Pinel Martínez, « Negative Theology and its Interpretation as an Atheistic Attitude in $5^{\text {th }}$ Century Athens », montre qu'il existe probablement un lien entre la montée des idées athées à la fin du $\mathrm{V}^{\mathrm{e}}$ siècle av. $\mathrm{n}$. è., et la tradition d'une théologie négative. Les écrits de Xénophane de Colophon, en effet, offrent les prémices non seulement d'une théologie négative, mais aussi d'un scepticisme épistémologique et théologique. Ces deux tendances auraient alors permis l'apparition du doute quant à l'existence des dieux, ce dont témoignent les écrits de Protagoras. Cet agnosticisme aurait, à son tour, cédé le pas à l'apparition d'un athéisme positif.

Enfin, Stéphanie Anthonioz, «Vision, interdit de représentation et via negationis dans la Bible hébraïque », s'intéresse à la dimension visuelle / matérielle et conceptuelle de la théologie négative à travers les visions bibliques, en particulier l'interdit de la représentation divine qui développe le premier commandement du décalogue (Ex 20,2-6; Dt 5,6-10), dans son contexte littéraire et historique. Les conclusions de ce travail amènent à affiner la compréhension de cet interdit et à saisir dans une approche historique mais aussi culturelle et sociale non pas les prémices, mais déjà une via negationis, la via negationis de la représentation divine. Cette contribution est placée en fin de dossier, car si elle rejoint des enjeux sémitiques, les deux contributions « grecques » qui la précèdent offrent un éclairage inattendu sur les notions abordées de « forme », d' « idée » et de « critique ».

Ainsi, les sources antiques, dans la diversité de leurs genres, présentent non pas - on l'a compris - une voie systématisée, mais les prémices d'une théologie négative. Ces premiers éléments montrent que le discours sur le sujet divin est le lieu d'une réflexion intense et d'interrogations multiples et la via negationis est envisagée comme un recours possible sinon nécessaire. Elle est déjà le corollaire de nombreuses représentations / discours sur le divin, et devient, en vertu de l'usage théologique qui peut en être fait, une voie nouvelle pour explorer le divin, et avant tout pour souligner les limites mêmes du langage, la tension voire la contradiction entre le sujet humain et son objet. À la question posée «Pourquoi dire le divin par la négation plutôt que par l'affirmation, puisque c'est de fait encore discourir sur le divin ? », il semble que trois éléments de réponse se font jour 
dans les sources antiques: considérer qu'il existe un principe vivant autre qu'humain, considérer que ce principe est plus grand que l'humain, mais qu'il est donné à ce dernier de s'en approcher, même imparfaitement.

stephanie.anthonioz@univ-catholille.fr sebastian.fink@uibk.ac.at 